\title{
Fatores direcionadores da cooperação de pequenas e médias empresas com a universidade: evidências a partir de quatro estudos de caso*
}

\author{
Vanessa Parreiras Oliveira ${ }^{\dagger}$ \\ Renato Garcia \\ Miguel Juan Bacic ${ }^{\S}$
}

\begin{abstract}
Resumo
Este artigo traz novos elementos para a temática da cooperação entre universidades e institutos de pesquisa e empresas por meio da investigação dos fatores direcionadores (drivers) dos relacionamentos cooperativos de pequenas e médias empresas. Para isso, explora os resultados de quatro estudos de caso de relacionamentos cooperativos de pequenas empresas com universidades e institutos de pesquisa no Brasil, os quais mostram que a capacidade de absorção da empresa, a existência de vínculos prévios e as proximidades geográfica e cognitiva entre os agentes são importantes fatores direcionadores do estabelecimento e do desenvolvimento das relações de colaboração. Ademais, evidenciam que a elevada capacidade de absorção da empresa e a proximidade cognitiva entre os agentes podem estimular o estabelecimento de relações de cooperação a maiores distâncias geográficas. Os resultados mostram, ainda, a importância do acesso ao financiamento público para a cooperação, especialmente quando compreendem maior complexidade e risco.
\end{abstract}

Palavras-chave: cooperação universidade-empresa, pequenas e médias empresas, fatores direcionadores, conhecimento e inovação

JEL: O31, O32, O33, L26

\section{Introdução}

Há elevado reconhecimento na literatura sobre a importância da cooperação com universidades para as atividades inovativas das firmas. Diversos estudos mostraram que as

\footnotetext{
*Aos pesquisadores e instituições que contribuíram para elaboração deste estudo.

${ }^{\dagger}$ Doutoranda do Programa de Pós-graduação em Teoria Econômica, Instituto de Economia, Universidade Estadual de Campinas. E-mail: vparreiras@uol.com.br.

${ }^{\ddagger}$ Professor do Instituto de Economia, Universidade Estadual de Campinas. E-mail: renatogarcia@eco.unicamp.br.

${ }^{\S}$ Professor do Instituto de Economia, Universidade Estadual de Campinas. E-mail: bacic@unicamp.br.
} 
pequenas empresas se beneficiam mais dos spillovers da pesquisa realizada na universidade dos que as grandes empresas, mais aptas a se apoiarem nos seus esforços internos de P\&D (LINK; REES, 1990; ACS; AUDRETSCH; FELDMAN, 1994a, 1994b; AUDRETSCH; VIVARELLI, 1994). Estudos pioneiros apontam que as grandes firmas apresentaram menor produtividade em P\&D do que as pequenas empresas e são, por isso, menos eficientes em explorar os benefícios derivados das interações com as instituições de pesquisa (LINK; REES, 1990; ACS; AUDRETSCH; FELDMAN, 1994a). Enquanto os dispêndios em P\&D da firma contribuem para a geração do resultado inovativo a empresas de portes diversos, os spillovers da universidade são mais importantes para a inovação das pequenas empresas (AUDRETSCH; VIVARELLI, 1994). Além disso, evidências empíricas indicam que os spillovers de universidades e laboratórios de pesquisa são geograficamente restritos (ACS; AUDRETSCH; FELDMAN, 1994a).

Existem controvérsias no que se refere ao efeito do tamanho da firma sobre a probabilidade de cooperar com a universidade. As empresas grandes, de um lado, são capazes de dedicar maiores recursos e tempo à construção de vínculos com as universidades (FONTANA; GEUNA; MATT, 2003; CARDAMONE; PUPO, 2015). As maiores firmas são mais propensas a possuir a capacidade para explorar fontes externas de conhecimento e para gerir interações com universidades, devido às grandes firmas serem capazes de dedicar maiores recursos e tempo à construção de vínculos com universidades do que as pequenas firmas, que podem operar em um ambiente com recursos mais limitados (LAURSEN; SALTER, 2004). As grandes firmas são também mais propensas a empregarem um staff com treinamento profissional em ciência e engenharia. Com tal background profissional, esses funcionários são capazes de se beneficiarem de suas relações com universidades para dar suporte ao trabalho na organização. Por outro lado, as empresas menores possuem menos recursos internos e necessitam de mais conhecimento externo para seus esforços inovativos, o que se traduz em maior número de parceiros cooperativos (FONTANA; GEUNA; MATT, 2003; CARDAMONE; PUPO, 2015).

Inserido nesse debate, este trabalho traz novos elementos para essa discussão, por meio da investigação dos fatores direcionadores (drivers) dos relacionamentos cooperativos de pequenas e médias empresas (PMEs) com universidades e institutos de pesquisa. Para isso, explora os resultados de quatro estudos de caso ilustrativos de relacionamentos cooperativos de PMEs brasileiras com universidades. Deste modo, este artigo ajuda a preencher uma lacuna acerca da compreensão dos padrões de interação entre PMEs e universidades e institutos de pesquisa, evidenciando as características estruturais das empresas, seus relacionamentos cooperativos e o sistema nacional de inovação conducentes à cooperação universidade-empresa (U-E).

O texto está organizado em cinco seções, incluindo esta introdução. A segunda seção apresenta uma breve revisão da literatura empírica sobre a cooperação U-E, com foco em alguns dos principais direcionadores (drivers) do envolvimento das empresas com essa cooperação. A terceira seção aborda os procedimentos metodológicos utilizados e o 
arcabouço analítico adotado na análise dos resultados. A quarta seção expõe a discussão dos resultados de pesquisa, seguida pelas considerações finais na quinta seção.

\section{Direcionadores (drivers) da cooperação entre empresas e universi- dades}

Estudos empíricos identificaram as principais variáveis que influenciam a propensão de as firmas cooperarem com universidades. Da perspectiva daquelas, destacam-se fatores estruturais, comportamentais, geográficos e relacionados a políticas como os mais importantes impulsionadores da cooperação entre organizações públicas de pesquisa e a indústria (FUENTES; DUTRÉNIT, 2012).

A habilidade de uma firma reconhecer o valor da nova informação externa, assimilála e aplicá-la a fins comerciais é crítica para suas capacidades inovativas (COHEN; LEVINTHAL, 1990). Sua capacidade de absorção é, em grande medida, uma função do nível de conhecimento anterior relacionado da firma. Uma corrente da literatura investigou o impacto da capacidade de absorção da empresa sobre a cooperação U-E e identificou que a falta de capacidade de absorção pode constituir uma significativa barreira ao relacionamento cooperativo. As firmas com empregados altamente qualificados são as que mais colaboram com universidades (BRUNEEL; D'ESTE; SALTER, 2010; LAURSEN; REICHSTEIN; SALTER, 2011; GARCIA et al., 2015; DREJER; ØSTERGAARD, 2017). Em relação aos determinantes das dimensões da capacidade de absorção, a capacidade de adquirir o conhecimento externo não está relacionada com a atividade de P\&D interna (TEIXEIRA et al., 2016), visto que se relaciona ao predomínio de trabalhadores com pós-graduação, que podem agir como gatekeepers do conhecimento, facilitando a identificação e a aquisição desse conhecimento na ausência de esforços mais estruturados de P\&D na empresa.

Em muitos casos, é possível observar que relacionamentos cooperativos alcançariam melhores resultados se os parceiros tivessem tido experiências cooperativas prévias. Os vínculos prévios constituem um dos fatores contextuais e organizacionais mais destacados relativos ao sucesso de acordos cooperativos entre firmas e universidades (MORAVALENTIN; MONTORO-SANCHEZ; GUERRAS-MARTIN, 2004). Acordos de cooperação mais bem-sucedidos são aqueles inseridos em relacionamentos cooperativos prévios, especialmente quando a colaboração passada gerou resultados positivos para os parceiros envolvidos. Os relacionamentos pessoais exercem papel muito relevante (LEMOS; CARIO, 2015), particularmente no que tange à existência de vínculos prévios como um fator direcionador do estabelecimento da cooperação U-E, os quais são importantes não apenas para o estabelecimento dos primeiros contatos com os potenciais parceiros de pesquisa, mas também para que os relacionamentos possam se firmar.

Diferentes padrões de apoio financeiro também influenciam o estabelecimento e a 
frequência das colaborações U-E (FUENTES; DUTRÉNIT, 2012). Diversos estudos prévios investigaram o impacto das políticas de Ciência, Tecnologia e Inovação (CT\&I) e industrial sobre a cooperação U-E, os quais identificaram que o apoio governamental aumenta a incidência de exploração de conhecimento de universidades/laboratórios governamentais e o estabelecimento de colaborações diretas em inovação com eles (MOHNEN; HOAREAU, 2003). A natureza do financiamento da cooperação parece não interferir nas fontes de informação e nos modos de interação, mas sim nos objetivos (ou na motivação) da cooperação U-E (RAPINI; OLIVEIRA; SILVA-NETO, 2013). Em geral, é positiva a avaliação dos impactos de programas da política de CT\&I e industrial sobre a cooperação U-E no Brasil (SALLES-FILHO, 2011; TEIXEIRA; MENEZES, 2013; CARRIJO; BOTELHO, 2013; SALLES-FILHO et al., 2012).

A literatura de inovação sublinha o papel das relações pessoais e da proximidade geográfica para a colaboração. Frequentemente, argumenta-se que quanto mais proximidade há entre os atores, maior é sua interação, seu aprendizado e sua inovação (BOSCHMA, 2005). Essa proximidade é particularmente importante caso a colaboração envolva o compartilhamento de conhecimento específico e tácito, que requer contato frequente face a face e mobilidade profissional (BISHOP; D'ESTE; NEELY, 2011); além disso, a proximidade geográfica com instituições públicas de pesquisa influencia a probabilidade de as firmas colaborarem com essas instituições (BROSTRÖM, 2010; GARCIA et al., 2011; LAURSEN; REICHSTEIN; SALTER, 2011), sugerindo que facilita os efeitos de spillovers. As firmas e as universidades tendem a co-localizar, resultando em uma grande proporção de firmas com ao menos uma universidade em uma curta distância (LAURSEN; REICHSTEIN; SALTER, 2011). No Brasil, parcela significativa das interações U-E no Brasil ocorre no mesmo espaço geográfico (GARCIA et al., 2011): a presença de grupos de pesquisa acadêmica na região, a localização de atividades inovativas nas empresas e a diversificação da estrutura produtiva local são fatores que estimulam as interações U-E.

Os vínculos entre agentes geograficamente próximos são mais prováveis de gerarem impulsos à inovação e criarem significativos efeitos de aprendizado na firma do que os vínculos distantes (BROSTRÖM, 2010). Ademais, a interação em proximidade geográfica é mais provável de contribuir, de maneira bem-sucedida, para projetos de P\&D a mercado no curto prazo; para os de longo prazo, a proximidade geográfica é, de modo geral, um fator menos crítico. Como regra, as firmas buscam novo conhecimento em grande proximidade à sua base de conhecimento existente, o que proporciona oportunidades e define as limitações para melhorias adicionais. A distância cognitiva não deve ser demasiadamente elevada, visto que a capacidade de os atores ou firmas absorverem novo conhecimento requer proximidade cognitiva, ou seja, sua própria base cognitiva deve ser próxima o suficiente ao novo conhecimento com a finalidade de comunicar, entender e processá-lo com sucesso (BOSCHMA; LAMBOOY, 1999; BOSCHMA, 2005).

No que se refere a outras dimensões não espaciais da proximidade, a proximidade cognitiva pode substituir a geográfica na colaboração U-E, já que a capacidade e a ex- 
pertise compartilhadas entre a firma que colabora e o parceiro acadêmico estimulam uma colaboração a longa distância (GARCIA, 2016). As relações direcionadas pelos empregados (medidas pelo local de qualificação e sua disciplina científica) e pelos altos gerentes influenciam fortemente a probabilidade de que as firmas colaborem com universidades específicas (DREJER; ØSTERGAARD, 2017). Esse resultado confirma a existência de efeitos separados e sobrepostos das relações direcionadas pelos empregados e da proximidade geográfica.

\section{Metodologia}

Este trabalho utilizou o método de estudos de caso ilustrativos com a finalidade de elucidar os fatores direcionadores dos relacionamentos cooperativos U-E de PMEs e proporcionar novas evidências de pesquisa. Trata-se de um estudo descritivo-explicativo de abordagem qualitativa, cujos meios de investigação utilizados foram a pesquisa bibliográfica e documental e o levantamento de campo.

A escolha por estudos de caso considerou informações provenientes do CNPq e buscas realizadas nos sítios da Inova - Agência de Inovação da Universidade Estadual de Campinas (UNICAMP), e da Coordenadoria de Transferência e Inovação Tecnológica (CTIT) da Universidade Federal de Minas Gerais (UFMG). Foram selecionadas para investigação detalhada duas pequenas e duas médias empresas brasileiras, segundo classificações de porte do Serviço Brasileiro de Apoio às Micro e Pequenas Empresas (SEBRAE) e do Banco Nacional de Desenvolvimento Econômico e Social (BNDES).

A pesquisa de campo foi conduzida entre maio de 2015 e julho de 2017. As quatro PMEs selecionadas, caracterizadas a seguir, foram objeto de visitas, oportunidades em que foram realizadas entrevistas semiestruturadas com proprietários, Chief Executive Officers (CEOs) ou colaboradores que integram ou se relacionam com as atividades da área de PD\&I. Foram realizadas também entrevistas semiestruturadas com seis pesquisadores de universidades com os quais as PMEs estabeleceram relacionamentos cooperativos.

\subsection{Empresas selecionadas}

\subsubsection{Griaule}

A Griaule é uma empresa em processo de crescimento que atua no desenvolvimento e licenciamento de programas de computador não-customizáveis, especializada em tecnologias biométricas, notadamente no reconhecimento de impressões faciais e digitais para uso em aplicações convencionais ou em segurança pública.

A opção estratégica da empresa é basear seus negócios no desenvolvimento de softwares e na venda para distribuidores; para isso, desenvolve componentes de softwares para organizações que os integram a um produto, chamadas empresas integradoras (por exem- 
90 Fatores direcionadores da cooperação de pequenas e médias empresas com a universidade: evidências a partir de quatro estudos de caso

plo, Diebold e Oracle), responsáveis por formatar o produto de acordo com as necessidades de seus clientes: pequenos estabelecimentos, grandes corporações e governos.

A despeito da abrangência internacional de sua atuação, em maio de 2015, a empresa possuía 25 funcionários que atuam nas sedes administrativa e de $\mathrm{P} \& \mathrm{D}$, localizadas nas proximidades do campus da UNICAMP, além de um escritório comercial em San José, na Califórnia. No ano de 2014, o faturamento da empresa foi da ordem de R \$ 34 milhões.

A Griaule foi criada na Incamp, incubadora da UNICAMP, no ano de 2002, tendo saído do período de incubação em 2005. Segundo o CEO e cientista-chefe, na fase de incubação, obteve acesso à estrutura da UNICAMP, notadamente a pesquisadores altamente qualificados e à oportunidade de capacitação para o empreendedorismo e para a gestão de empresas. Todavia, o principal benefício da incubação apontado foi a associação desta à marca da universidade.

Atualmente, a Griaule mantém com a UNICAMP um vínculo contratual, por meio do qual utiliza a marca de empresa graduada pela incubadora da universidade, em razão de no período pós-incubação, os principais canais de transferência de conhecimento serem a contratação de recém-graduados e pós-graduados, e a troca informal de informações entre os recursos humanos da empresa alocados no departamento interno de P\&D e seus ex-professores da Faculdade de Engenharia Elétrica e de Computação (FEEC).

\subsubsection{Bio Brasil Biotecnologia}

A Bio Brasil Biotecnologia é uma fabricante de materiais para medicina e odontologia a qual possuía, em novembro de 2015, 25 funcionários. Resultado da fusão de dois grupos de investidores, foi fundada em 2009, no Polo Industrial de Anápolis, e possui uma unidade em Vinhedo, região metropolitana de Campinas.

Para iniciar suas atividades, a empresa assumiu a produção e comercialização de dois produtos. O primeiro é o TF-TEST, kit para exame copro-parasitológico, objeto de licenciamento de tecnologia com a UNICAMP; para tanto, adquiriu a Immunoassay Indústria e Comércio, empresa que pertencia a um dos grupos de investidores e que participou do desenvolvimento da tecnologia do TF-Test ao lado de pesquisadores da UNICAMP e da Universidade de São Paulo (USP). O segundo contempla a linha da Família Clean, voltada à segregação e ao descarte de resíduos médico-hospitalares.

O desenvolvimento do TF Test compreendeu, no início da década de 2000, uma cooperação científica multidisciplinar com três pesquisadores (um da USP e dois da UNICAMP), que idealizaram e desenharam um kit para o diagnóstico de parasitoses gastrointestinais de humanos em amostras fecais (GOMES; HOSHINO-SHIMIZU; FALCAO, 2008). Os pesquisadores da área biológica perceberam a importância de atrelar as áreas de Biologia, Ciência da Computação, Ciências Farmacêuticas e Engenharia Industrial para desenvolvimento desse sistema por análise computadorizada de imagens e de seu uso, intitulado DAPI - Diagnóstico Automatizado de Parasitos Intestinais (INOVA UNI-

Econômica-Niterói, v. 20, n. 2, p. 85-106. Dezembro, 2018 
CAMP, 2011). Por meio desse novo sistema de diagnóstico, o computador obtém detecção automática de parasitos intestinais (ALVES-FILHO, 2007).

A opção estratégica da Bio Brasil Biotecnologia foi a externalização de suas atividades de P\&D para a UNICAMP. Para internalizar a produção e nacionalizar as tecnologias, a empresa adquiriu patentes e linhas de produtos internacionais, logo mantém a parceria com a UNICAMP, advinda da adquirida Immunoassay Indústria e Comércio.

\subsubsection{Padtec}

A Padtec é uma empresa voltada ao desenvolvimento, à fabricação e à comercialização de soluções turnkey para sistemas ópticos: produtos de alta tecnologia para transmissão de dados em redes de fibra óptica. Os sistemas de comunicações ópticas baseados na tecnologia DWDM (Dense Wavelength Division Multiplexing), utilizados no núcleo da rede, possuem aplicações em redes de entroncamento, como de longa distância, metropolitanas ou de conexões ponto a ponto em redes de armazenamento.

A Padtec foi constituída, no ano de 2001, como uma cisão da Fundação CPqD-Centro de Pesquisa e Desenvolvimento em Telecomunicações (PADTEC, 2017). Com sede em Campinas, está localizada no Polo de Alta Tecnologia de Campinas, dentro das instalações do parque industrial do $\mathrm{CPqD}$. A empresa tem no setor de telecomunicações seu principal mercado; outrossim, a procura por soluções DWDM tem estimulado seus negócios da empresa, visto que, além das grandes operadoras, os provedores de internet e de serviços over-the-top (OTTs), os centros estaduais de processamento de dados e as concessionárias de energia elétrica com redes OPGW também contribuíram para o aumento de sua demanda no período recente (PADTEC, 2017). A Padtec destaca-se também pela presença nas redes de entroncamento dos maiores provedores de serviços de telecomunicações da América Latina (PADTEC, 2017). É a única empresa no hemisfério sul desenvolvedora de tecnologia DWDM.

Ao final dos exercícios de 2012 e 2011, a empresa possuía 387 e 349 funcionários, e apresentou faturamento da ordem de R \$ 273 e 286 milhões, respectivamente. Em 2013, ano em que apresentou um crescimento acentuado, contou com mais de 500 colaboradores e auferiu receita bruta consolidada de $\mathrm{R} \$ 390$ milhões (PADTEC, 2017). No ano de 2016, alcançou a receita bruta de $\mathrm{R} \$ 334$ milhões e, em janeiro de 2017, empregava 595 funcionários.

A Padtec caracteriza-se por realizar esforços importantes em inovação tecnológica e, em especial, em P\&D. Com a finalidade de se manter na vanguarda da tecnologia e ofertar soluções de elevado valor agregado, fomenta, por meio de convênios, atividades de P\&D por meio de centros de excelência em comunicações ópticas. Dois centros bastante reconhecidos em comunicações ópticas - o CPqD e a UNICAMP -, realizam, em parceria, os trabalhos de desenvolvimento tecnológico mais próximos à fronteira do conhecimento e, portanto, sujeitos a maior risco (PADTEC, 2017). 
92 Fatores direcionadores da cooperação de pequenas e médias empresas com a universidade: evidências a partir de quatro estudos de caso

A empresa estabeleceu relacionamentos cooperativos com grupos de pesquisa das seguintes universidades e institutos de pesquisa: $\mathrm{CPqD}$; Universidade Federal de Pernambuco - UFPE; Universidade Federal de Itajubá - UNIFEI; UNICAMP; Universidade Estadual de Ceará - UECE; Centro Nacional de Pesquisa em Energia e Materiais - CNPEM; Universidade Estadual Paulista Júlio de Mesquita Filho - UNESP e Instituto Atlântico. Segundo os entrevistados, os relacionamentos cooperativos U-E da Padtec referem-se a projetos distintos, com diferentes objetivos. As pesquisas com considerações de uso imediato de resultados são o modelo de relacionamento U-E mais utilizado pela empresa pela relevância para a sua estratégia.

\subsubsection{Seva}

A Seva Engenharia Eletrônica S.A é uma empresa de capital fechado, sediada no Distrito Industrial de Contagem, região metropolitana de Belo Horizonte. Em agosto de 2016, empregava 220 funcionários, e tem experimentado crescimento, tanto em termos de número de empregados quanto de faturamento: em meados de 2010, possuía 85 funcionários; em 2014, seu faturamento bruto foi da ordem de $\mathrm{R} \$ 31$ milhões.

A Seva foi fundada no ano de 1993, com foco no desenvolvimento de tecnologia e na fabricação de dispositivos eletrônicos, demonstrando-se bastante bem-sucedida na área. No ano de 2001, assumiu um importante papel no desenvolvimento de sistemas de telemetria e de controle de frotas, posicionando-se como uma importante geradora de tecnologia em monitoramento do país. A empresa pode ser definida como "intensiva em engenharia", cujo principal ativo é a capacidade de engenharia, especialmente elétrica e eletrônica. Fundamentada em desenvolver tecnologias para o mercado automobilístico, atualmente apresenta um caráter multidisciplinar, com aplicações de Internet of Things IOT nas áreas de automobilística/automotiva, saúde, agronegócio, controle de processos e energia alternativa.

A empresa possui equipe própria e permanente de desenvolvimento. Em sua estratégia inovativa, desenvolve projetos tecnológicos com participação de áreas multidisciplinares, pesquisas de aplicação de novos conhecimentos e parcerias com empresas, universidades e centros de pesquisas, como Embrapa. Além disso, a Seva estabeleceu relacionamentos cooperativos (formais ou informais) com pesquisadores do Instituto de Ciências Biológicas (ICB) da UFMG; Instituto de Ciências Exatas (ICEx) da UFMG); Escola de Veterinária da UFMG; Departamento de Ciência da Computação (DECOM) da UFOP; e ESALQ/USP, UNESP, Universidade Federal de Viçosa - UFV e Fazu - Faculdades Associadas de Uberaba, entre outras. Algumas dessas parcerias ocorreram por meio da controlada Intergado, marca com a qual a Seva atua no desenvolvimento de equipamentos de zootecnia de precisão.

Econômica-Niterói, v. 20, n. 2, p. 85-106. Dezembro, 2018 


\subsection{Os fatores direcionadores da cooperação de PMEs com universidades e institu- tos de pesquisa: um arcabouço analítico}

$\mathrm{O}$ arcabouço analítico adotado baseia-se em um conjunto de fatores estruturais, comportamentais, geográficos e relacionados a políticas apontados pela literatura como direcionadores (drivers) do estabelecimento e desenvolvimento de relacionamentos cooperativos U-E.

1. A capacidade de absorção da firma A literatura identifica que a qualificação da mão de obra e as atividades internas de P\&D são importantes determinantes da capacidade de absorção da firma. Na investigação dessa capacidade nas quatro PMEs que cooperam com universidades e institutos de pesquisa, este trabalho examinou suas atividades inovativas (atividades internas de P\&D e aquisição externa de P\&D e de outros conhecimentos externos) e a qualificação da sua mão de obra.

2. A existência de vínculos (links) prévios entre as partes

Vínculos (links) prévios podem ser definidos quando, no passado, houve alguma colaboração em atividades similares ou aos parceiros colaborarem previamente em outras ocasiões (MORA-VALENTIN; MONTORO-SANCHEZ; GUERRASMARTIN, 2004). Para investigar se a existência de vínculos prévios entre as PMEs e as universidades constituíram um fator direcionador da cooperação U-E, verificouse, particularmente, se havia vínculos prévios dos indivíduos-chave no estabelecimento da cooperação com pesquisadores das respectivas universidades, bem como a natureza dessas conexões.

3. A existência de indivíduos na firma que se comportam como "gatekeepers do conhecimento"

A capacidade de absorção depende dos indivíduos que permanecem na interface da firma e do ambiente externo ou da interface entre subunidades no interior da firma (COHEN; LEVINTHAL, 1990). Quando a expertise da maioria dos indivíduos na organização difere consideravelmente daquelas dos atores externos que provêm informação útil, alguns membros dos grupos provavelmente assumem papéis relativamente centralizados de "gatekeeping" ou "boundary-spanning".

Na busca de evidências da existência de indivíduos nas PMEs que as nutrem com conhecimento absorvido de fontes externas, comportam-se como "gatekeepers do conhecimento", consequentemente propiciam o estabelecimento e o desenvolvimento da cooperação U-E (ALLEN, 1977). Sua características principais são: a) constituem uma pequena comunidade de indivíduos - os gatekeepers são um pequeno número de pessoas-chave para quem os outros frequentemente recorrem para informação e diferem dos seus colegas no grau para o qual eles expõem a si próprios a fontes de informação tecnológica fora de sua organização; b) estão no núcleo de 
94 Fatores direcionadores da cooperação de pequenas e médias empresas com a universidade: evidências a partir de quatro estudos de caso

uma rede de informações; c) expõem-se demasiadamente a fontes externas de informação; e d) suas ligações com atores externos são principalmente informais. Adicionalmente, os gatekeepers podem entender ao menos uma porção do material publicado em periódicos científicos, a fim de traduzir a informação em termos nos quais os tecnologistas médios possam usar, o que implica também desempenhar uma função de "transcodificação" para aqueles (outros atores na organização) que não podem interagir com fontes externas de conhecimento (ALLEN, 1977).

4. O acesso ao financiamento público e a concessão de benefícios fiscais

Neste trabalho, analisou-se o impacto do acesso de PMEs a diferentes instrumentos de financiamento público no estabelecimento da cooperação U-E. Além disso, foram investigados os impactos da concessão dos benefícios fiscais, sobretudo no âmbito da Lei de Informática.

5. A proximidade geográfica e proximidade cognitiva entre os agentes

$\mathrm{Na}$ investigação do papel desempenhado pelas proximidades geográfica e cognitiva entre os agentes no estabelecimento e no desenvolvimento da colaboração U-E, foram tomadas como referência, primeiramente, as distâncias geográficas em linha reta entre as quatro PMEs e faculdades ou institutos de universidades com os quais elas estabeleceram-se relacionamentos cooperativos. Em segundo lugar, procurouse identificar a existência de um background disciplinar comum, isto é, uma base de conhecimento compartilhada pelos agentes envolvidos na cooperação.

\section{Discussão dos resultados}

\subsection{A capacidade de absorção da firma}

Os resultados alcançados evidenciaram que as atividades internas de $\mathrm{P} \& \mathrm{D}$ e a qualificação da mão de obra das empresas Griaule, Padtec e Seva favorecem o desenvolvimento da sua capacidade de absorção. Essas empresas realizam atividades internas de P\&D formais e contínuas, além de possuírem recursos humanos qualificados alocados nessas atividades. As atividades internas de $\mathrm{P} \& \mathrm{D}$ destas foram caracterizadas pelos entrevistados como "importante" para que inovem. Essas empresas identificam-se também devido à significativa participação de profissionais com nível superior ou mais no conjunto dos seus recursos humanos.

A Padtec realiza consistentes esforços de busca de conhecimento externo, para assimilálo (inclusive de fontes de conhecimento externas localizadas no exterior) e incorporá-lo aos seus produtos. Suas atividades internas de $\mathrm{P} \& \mathrm{D}$ são contínuas e conduzidas por uma equipe composta, em janeiro de 2017, por 90 profissionais de nível superior, formada principalmente por engenheiros elétricos e de computação $(15,1 \%$ dos recursos humanos), físicos e graduados em ciência da computação. A participação de pós-graduados

Econômica-Niterói, v. 20, n. 2, p. 85-106. Dezembro, 2018 
(mestres e doutores) na Diretoria de Tecnologia da empresa varia entre 35 e $40 \%{ }^{1}$. Além dos esforços internos de P\&D, a Padtec Submarine, unidade de negócios da empresa voltada para comunicação óptica por cabos submarinos, é composta por uma equipe de pesquisadores/desenvolvedores na Itália e um back office no Brasil (PADTEC, 2017). A unidade italiana é contemplada por consultores com notório conhecimento em comunicações ópticas submarinas, contratados para atuarem no desenvolvimento de equipamentos $^{2}$. Os dispêndios totais em P\&D alcançam o patamar de aproximadamente $15 \%$ do faturamento anual da empresa, percentual mantido estável, o que demonstra a relevância das atividades dessa área na estratégia da empresa.

No caso da Seva, a empresa possuía, em agosto de 2016, uma equipe interna de P\&D e engenharia, que contava com aproximadamente 50 engenheiros em dedicação integral (22\% dos recursos humanos), por atribuir elevada importância às atividades inovativas: entre $15 \%$ e $20 \%$ de seu faturamento bruto são dedicados a PD\&I.

Já a Bio Brasil Biotecnologia evidenciou que a capacidade de absorção da organização está centrada em um indivíduo, o gerente de produtos da empresa (egresso da UNICAMP), por meio do qual a organização explora as fontes externas de conhecimento e realiza a gestão dos projetos de cooperação com as universidades parceiras. Ainda que seja detentora de patentes nacionais e internacionais referentes à tecnologia DAPI, ela não possui infraestrutura própria de $\mathrm{P} \& \mathrm{D}$ em suas unidades em Vinhedo e Anápolis. Todos seus recursos humanos dedicados às atividades de $\mathrm{P} \& \mathrm{D}$ desenvolvem-nas no interior do Instituto de Computação (IC) da UNICAMP. Nesse caso, infere-se que a colaboração com a universidade ocorre na ausência de esforços internos de pesquisa na empresa.

\subsection{A existência de indivíduos na firma que se comportam como "gatekeepers do conhecimento"}

A existência de indivíduos que nutrem a pequena empresa com conhecimento absorvido de fontes externas configura a ocorrência de "gatekeepers do conhecimento", capazes de encontrar, estabelecer e gerir os projetos de cooperação dessas organizações com as universidades. Sua importância é evidenciada nos casos da Bio Brasil Biotecnologia e da Seva, especialmente nas operações da Intergado. Na primeira empresa, o gerente de produto e ex-aluno do Instituto de Biologia (IB) da UNICAMP, com graduação em Biologia e mestrado em Parasitologia, exerce o relevante papel de vincular sua empresa à universidade. De fato, esse agente: a) está no núcleo de uma rede de informações que engloba pesquisadores universitários da UNICAMP e da USP; da Bio Brasil Biotecnolo-

\footnotetext{
${ }^{1}$ A maior área da Padtec é a de operações: em janeiro de 2017, era composta por aproximadamente 300 funcionários, com participação majoritária da equipe de campo, em sua maior parte, profissionais de nível técnico.

${ }^{2}$ A empresa realizou a transferência da tecnologia entre as equipes italiana e brasileira para o desenvolvimento do equipamento repetidor óptico submarino para a Google. Dessa forma, procurava replicar, internamente, a tecnologia desenvolvida na Itália.
} 
96 Fatores direcionadores da cooperação de pequenas e médias empresas com a universidade: evidências a partir de quatro estudos de caso

gia, que realizam seus trabalhos no IC/UNICAMP, através dos projetos colaborativos; e a empresa na qual circulam informações sobre os desenvolvimentos referentes ao TF-Test e TF-Test Modified; b) está muito exposto às fontes externas de informação, em permanente e intensa interação com a equipe de pesquisadores sediada na UNICAMP, ou seja, pesquisadores da empresa que se dedicam a atividades de P\&D e da universidade; e c) possui vínculos, sobretudo informais, com a universidade.

Mesmo quando um agente que exerce o papel de gatekeeper é importante, sua capacidade de absorção individual não constitui a capacidade de absorção de sua unidade na firma (COHEN; LEVINTHAL, 1990). A facilidade ou dificuldade do processo de comunicação interna à firma e, por sua vez, o nível de capacidade de absorção organizacional não são somente uma função das capacidades do gatekeeper, mas também das capacitações daqueles a quem este transmite a informação. Portanto, contar com um pequeno conjunto de gatekeepers tecnológicos pode não ser suficiente, visto que o grupo todo deve ter uma base de conhecimento relevante; quando as estruturas de conhecimento são altamente diferenciadas, o nível requerido de conhecimento de base pode ser bastante elevado.

No caso da Intergado (Seva), também foi possível identificar dois ex-alunos da Escola de Veterinária da UFMG (com graduação e doutorado em Medicina Veterinária) que participavam do projeto para condução de pesquisas científicas, validação de equipamentos em zootecnia de precisão e realização de testes/experimentos com a universidade, na condição de bolsistas RHAE/CNPq. Na Seva, há também uma bióloga, com doutorado em Bioquímica e Imunologia pelo ICB/UFMG, que participa do projeto cooperativo com o ICEx/UFMG, voltado para o desenvolvimento de um biosensor à base de grafeno para testes diagnósticos.

\subsection{A existência de vínculos (links) prévios entre as partes}

Os resultados da pesquisa indicam que a existência de vínculos prévios entre os parceiros constituiu um importante fator direcionador do estabelecimento e do desenvolvimento dos projetos colaborativos com a universidade. Nesses casos, indivíduos-chave, particularmente ex-alunos de graduação e pós-graduação, que já possuíam contatos prévios com pesquisadores das universidades, tornaram-se um importante fator para o estabelecimento e a gestão dos projetos colaborativos.

Evidências mostram que os vínculos prévios de ex-alunos têm o efeito de retroalimentar os projetos de cooperação com a universidade. As contratações de ex-discentes desenvolvem, de modo cíclico, os relacionamentos cooperativos, particularmente quando se verifica também a proximidade geográfica entre os parceiros, visto que os pesquisadores da empresa comumente voltam a acessar o conhecimento de seus ex-professores e ex-colegas na universidade. Ademais, novas contratações de egressos de universidades específicas decorrem dos vínculos prévios estabelecidos entre as universidades e as

Econômica-Niterói, v. 20, n. 2, p. 85-106. Dezembro, 2018 
empresas em relacionamentos cooperativos.

A existência de vínculos prévios entre os parceiros foi um fator direcionador do estabelecimento e do desenvolvimento dos projetos de colaboração entre a UNICAMP e as empresas Griaule e Bio Brasil Biotecnologia, bem como das interações da Padtec com grupos de pesquisa da UNICAMP, do CPqD, da UNIFEI e da UFPE. No caso da Seva, os vínculos prévios também foram importantes direcionadores do relacionamento da Intergado com a Escola de Veterinária da UFMG. Entretanto, as empresas Griaule, Padtec e Seva evidenciaram que já desenvolveram capacitações para a busca de conhecimento externo (NELSON; WINTER, 2005; COHEN; LEVINTHAL, 1990), em razão de serem capazes de identificar o conhecimento externo relevante e estabelecer vínculos cooperativos, mesmo quando não há vínculos prévios entre os agentes.

O caso da Griaule mostra, desde a sua fundação, uma forte vinculação com a UNICAMP, o que se materializa sob duas formas: a) a condição de empresa graduada na incubadora da universidade (Incamp); e b) o número expressivo de ex-alunos de graduação e pós-graduação de engenharia de computação (95\% dos pesquisadores da empresa), que se dedicam exclusivamente às atividades de P\&D internas. Outro canal de interação entre a UNICAMP e a Griaule é a troca informal de informações entre seus pesquisadores e os professores da FEEC/UNICAMP.

$\mathrm{O}$ vínculo da Bio Brasil Biotecnologia com a UNICAMP remonta à empresa adquirida Immunoassay Indústria e Comércio, que, como indicado, era sócia controladora da ImmunoCamp Pesquisa e Desenvolvimento de Tecnologia, organização que esteve incubada na Incamp/UNICAMP; inclusive um dos pesquisadores da universidade, diretamente envolvido com o desenvolvimento da tecnologia DAPI, foi assessor científico da Immunoassay Indústria e Comércio.

Parcela significativa dos relacionamentos cooperativos da Padtec com as universidades também indicam a importância dos vínculos prévios, que também retroalimentam os projetos colaborativos. Na condição de spin-off, a Padtec possui vínculos estreitos com o $\mathrm{CPqD}$, fundação com a qual realiza os trabalhos de desenvolvimento tecnológico com maiores complexidade e risco. Ademais, desde a sua fundação, a empresa e pesquisadores do Instituto de Física Gleb Wataghin (IFGW) e da FEEC da UNICAMP possuem fortes vínculos cooperativos voltados ao desenvolvimento de tecnologias na área óptica e fotônica. A Padtec teve como cofundadores dois ex-alunos da UNICAMP (de graduação e pós-graduação) e parcela significativa dos profissionais alocados na $\mathrm{P} \& \mathrm{D}$ interna são egressos da UNICAMP (cerca de 50\%), o que demonstra a importância dos vínculos prévios para o estabelecimento dos projetos colaborativos com a universidade.

No caso da Seva, os mecanismos internos de busca e de identificação de potenciais parceiros na universidade demonstram o estreito relacionamento do CEO com instituições universitárias, devido a alguns fatores, como sua participação em diferentes conselhos da universidade, por meio dos quais consegue estabelecer contatos variados com a comunidade acadêmica. Há alguns projetos de colaboração com universidades nos quais a em- 
98 Fatores direcionadores da cooperação de pequenas e médias empresas com a universidade: evidências a partir de quatro estudos de caso

presa foi capaz de identificar potenciais parceiros, caso dos relacionamentos cooperativos da Seva com o ICB e o ICEx da UFMG, voltados, respectivamente, para desenvolvimento de uma solução para a dengue e de um biosensor à base de grafeno para testes diagnósticos. Além disso, a empresa é frequentemente procurada por pesquisadores acadêmicos para o estabelecimento de projetos de colaboração.

\subsection{A proximidade geográfica}

A proximidade geográfica entre as empresas investigadas e as universidades com relacionamentos cooperativos constitui um importante fator direcionador da maior parte desses relacionamentos, visto que essa proximidade facilita e estimula os processos de aprendizado interativo, o que se traduz na intensificação das formas de transferência do conhecimento gerado na universidade. Os resultados alcançados mostram a importância de dois canais de transferência de conhecimento nesses relacionamentos: troca informal de informações por meio de interações face-a-face e pesquisa conjunta. As interações pessoais diretas desenvolvem capital social, tal como confiança, "linguagem"conjunta e cultura de pesquisa conjunta (SCHARTINGER et al., 2002; GARCIA et al., 2011).

No caso da Griaule, a empresa localiza-se a cerca de $5 \mathrm{~km}$ da FEEC. A proximidade geográfica entre a empresa e a universidade constitui um importante direcionador da cooperação U-E, por facilitar as trocas informais de informação, um dos principais canais de transferência de conhecimento U-E presentes neste caso.

De maneira similar, a proximidade geográfica entre a Bio Brasil Biotecnologia - unidade Vinhedo e o IC/UNICAMP $(23 \mathrm{~km})$ é um importante fator direcionador do relacionamento cooperativo U-E, pois possibilita que o gerente de produto (e gatekeeper do conhecimento) mantenha intensas e frequentes trocas informais de informação com os pesquisadores do IC/UNICAMP por meio de encontros semanais.

A Padtec mantém alguns relacionamentos cooperativos com universidades e institutos de pesquisa em situação de proximidade geográfica, com contatos frequentes e trocas informais de informação: 1) o CPqD (a uma distância de cerca de 350 metros) e; 2) na UNICAMP, a FEEC e o IFGW (a 2,5 km) ${ }^{3}$. A maioria dos projetos de colaboração da Padtec de médio prazo, desenvolvedores de um novo produto, é realizada juntamente com o CPqD. Vale notar que a cooperação tecnológica da Padtec com o CPqD também incorpora pesquisas de curto prazo, com considerações de uso imediato dos resultados e prestação de serviços tecnológicos. De modo geral, os relacionamentos cooperativos da Padtec com a UNICAMP compreendem projetos de pesquisa aplicada, voltados ao desenvolvimento de produtos. Ademais, a universidade é comumente buscada pela empresa para a solução de problemas, situações em que a auxilia com equipamentos e recursos humanos.

\footnotetext{
${ }^{3}$ Ainda em condição de proximidade geográfica $(1,5 \mathrm{~km})$, a Padtec possui relacionamento cooperativo do tipo "Pesquisa científica com considerações de uso imediato de resultados" com o CNPEM.
}

Econômica-Niterói, v. 20, n. 2, p. 85-106. Dezembro, 2018 
No caso da Seva, a maior parte dos seus relacionamentos cooperativos ocorre em condições de proximidade geográfica, seja com a UFMG nos Departamentos de Parasitologia (ICB) e Física e Ciência da Computação (ICEx) e na Escola de Veterinária (a aproximadamente $8 \mathrm{~km}$ de distância), seja com a DECOM/UFOP $(80 \mathrm{~km})$

As evidências dos estudos de caso mostram que firmas com baixa capacidade de absorção dependem mais da proximidade geográfica com universidades, o que corrobora estudos prévios (GARCIA et al., 2015). Em contraste, as firmas com elevada capacidade de absorção possuem uma maior gama de parceiros acadêmicos potenciais, as quais são capazes de buscar (e encontrar) parceiros acadêmicos geograficamente mais distantes, o que reforça o papel da capacidade de absorção como um direcionador importante da colaboração das empresas com a universidade.

\subsection{A proximidade cognitiva}

Os quatro estudos de caso evidenciam que a proximidade cognitiva entre o pessoal de P\&D e de engenharia das empresas e os pesquisadores de universidades e institutos de pesquisa constitui um importante fator direcionador da cooperação U-E. Agentes com elevada capacidade cognitiva possuem uma base comum de conhecimento e expertise, $o$ que facilita a comunicação e fomenta processos de aprendizado interativo. O capital social constituído por meio das interações pessoais entre os agentes nas firmas e na universidade facilita a construção de laços de confiança entre eles (SCHARTINGER et al., 2002).

No caso da Griaule, a origem da empresa, na incubadora da UNICAMP, e as características de seu pessoal de pesquisa, cuja qualificação em engenharia foi realizada na FEEC, evidencia que a organização não precisa dedicar muitos recursos e tempo à construção de vínculos com a universidade, visto que esses vínculos foram construídos a partir de sua constituição e da composição de seus recursos humanos.

Na Bio Brasil, a formação acadêmica do gerente de produtos ocorreu na graduação em Biologia e mestrado em Parasitologia no IB/UNICAMP, o que promoveu a criação de laços comuns entre os o gatekeeper do conhecimento na empresa e os pesquisadores da universidade, denotando a forte proximidade cognitiva entre eles. Essa proximidade também se verifica em relação aos recursos humanos que desenvolvem as atividades de P\&D da empresa no IC/UNICAMP e os pesquisadores da universidade.

Em relação à Padtec, o compartilhamento de uma base de conhecimento comum pelos profissionais alocados na $\mathrm{P} \& \mathrm{D}$ interna e pelos engenheiros do $\mathrm{CPqD}$, bem como pelos pesquisadores da FEEC e do IFGW da UNICAMP, propicia que, na dinâmica dos relacionamentos cooperativos, sejam desenvolvidos projetos conjuntos de pesquisa e ocorram trocas informais de conhecimento entre as partes sobre questões específicas dos desenvolvimentos da empresa.

A proximidade cognitiva entre a Seva e seus parceiros acadêmicos também é evidenciada pela sua equipe de engenharia, composta por profissionais com formação bastante 
100 Fatores direcionadores da cooperação de pequenas e médias empresas com a universidade: evidências a partir de quatro estudos de caso

diversificada, que atuam em projetos específicos de colaboração, os quais são compostos por conhecimentos de caráter interdisciplinar.

\subsection{O acesso ao financiamento público e a concessão de benefícios fiscais no âmbito da Lei de Informática}

Primeiramente, é importante notar que todas as pequenas empresas investigadas são beneficiárias de programas públicos de financiamento a atividades inovativas de diversas agências, como FINEP - Subvenção e linhas de financiamento, BNDES - Subvenção, CNPq e agências estaduais: FAPESP e FAPEMIG. Em segundo lugar, os estudos de caso evidenciam que o financiamento público desempenhou um duplo papel como direcionador das cooperações U-E. Por um lado, o financiamento público foi o catalizador de alguns dos projetos de colaboração das empresas com as universidades parceiras, auxiliando-as a transporem a escassez interna de capital para esse fim, por meio do uso de recursos financeiros da agências de fomento e do compartilhamento de outros recursos, como pessoal e laboratórios, entre as empresas e as universidades envolvidas. Além disso, o financiamento público constituiu um fator direcionador fundamental de muitos desses projetos cooperativos, visto que foram compostos por recursos, financeiros e humanos (que as empresas não possuíam) e elevada complexidade e riscos.

Entre os casos analisados, há ao menos dois exemplos bastante precisos desse duplo papel do financiamento público. O primeiro é o desenvolvimento da nova tecnologia de exame parasitológico de TF Test e TF-Test Modified na empresa Bio Brasil, em que a ausência do esquema de financiamento público inviabilizaria o investimento necessário para o desenvolvimento da tecnologia. O segundo é o desenvolvimento do biosensor à base de grafeno para testes diagnósticos pela SEVA Engenharia: o volume de recursos e os riscos do processo também seriam fatores impeditivos para o incremento da tecnologia na empresa sem o uso de financiamento público ${ }^{4}$.

\footnotetext{
${ }^{4}$ Também na Seva, há um caso de insucesso no estabelecimento de um projeto de cooperação com o Departamento de Parasitologia do ICB/UFMG, voltado ao desenvolvimento de uma solução para a doença da dengue. Nesse caso, a ausência de acesso a recursos de subvenção inviabilizou o desenvolvimento da tecnologia devido a elevados montantes de recursos e riscos do processo. Inicialmente, a empresa apoiou um projeto de pesquisa em desenvolvimento no ICB, por meio de aporte de recursos próprios e de recursos obtidos com o SEBRAE e com suporte ao desenvolvimento na universidade. Contudo, um aporte de recursos por meio do sistema de subvenção da FINEP seria crucial para a continuidade do projeto de desenvolvimento da solução para a doença e para a sua viabilização em produto. Face à indisponibilidade do financiamento público de forma adequada, a empresa desistiu desse projeto e, em consequência, da parceria com a universidade.
}

Econômica-Niterói, v. 20, n. 2, p. 85-106. Dezembro, 2018 


\section{Considerações Finais}

Os resultados obtidos permitem identificar alguns fatores direcionadores que influenciam a decisão de as pequenas empresas cooperarem com a universidade. Esses resultados foram obtidos a partir da investigação de quatro casos de pequenas empresas que possuem projetos colaborativos com universidades no Brasil. Nesse sentido, a identificação desses fatores representa uma contribuição para o debate sobre as formas de colaboração entre a universidade e as pequenas empresas.

Um dos fatores identificados é a capacidade de absorção da firma, já que todos as empresas estudadas possuem em seus quadros diversos trabalhadores com elevado nível de qualificação. Em geral, as empresas também se caracterizam por realizar importantes esforços internos de $\mathrm{P} \& \mathrm{D}$, procedidos por profissionais qualificados e em dedicação exclusiva às atividades de $\mathrm{P} \& \mathrm{D}$. Esse conjunto de capacitações internas das firmas permite às empresas buscarem e explorarem as fontes externas de conhecimento, que se somam aos esforços internos de desenvolvimento de novas tecnologias. Em adição ao caso geral, uma das empresas analisadas realizou a opção de externalizar suas atividades de P\&D para a universidade; para isso, utiliza a infraestrutura de pesquisa da instituição para o desenvolvimento de tecnologias com potencial de serem utilizadas no rol de produtos da empresa.

Em casos como esse, deve-se mencionar a importância dos agentes que exercem o papel de gatekeepers do conhecimento, configurados como outro direcionador (driver) da cooperação entre as pequenas empresas e a universidade, em razão de estabelecerem contatos e estreitarem os vínculos com os pesquisadores acadêmicos, papel fundamental para o sucesso dos projetos de cooperação. Nesse contexto, foram observados outros fatores direcionadores da colaboração entre as pequenas empresas e a universidade, como a existência de vínculos prévios entre os agentes com essa função, os quais geralmente possuem algum tipo de vínculo prévio com os pesquisadores acadêmicos, especialmente com egressos da universidade, como ex-alunos ou ex-colegas. O papel desses vínculos prévios reveste-se de elevada importância, pois é capaz de estabelecer laços de confiança entre os parceiros, fundamentais para o sucesso da cooperação. A existência de desses vínculos e desses laços demonstrou-se fundamental para a gestão dos projetos de cooperação, inclusive porque permite minimizar potenciais conflitos entre os parceiros.

Além disso, não se deve subestimar a importância da proximidade geográfica e da proximidade cognitiva entre os parceiros. Em muitos casos, a proximidade cognitiva, definida pelo compartilhamento de expertise e de bases de conhecimento comuns entre os agentes, é estabelecida pela existência de vínculos prévios entre os agentes responsáveis pela cooperação. Essa proximidade facilita a comunicação e fomenta o processo de aprendizado interativo. Já a proximidade geográfica, verificada em muitos projetos colaborativos das empresas estudadas, também é um direcionador importante da cooperação, devido a viabilizar a interação frequente e os contatos face a face entre os parceiros, o que

Econômica-Niterói, v. 20, n. 2, p. 85-106. Dezembro, 2018 
102 Fatores direcionadores da cooperação de pequenas e médias empresas com a universidade: evidências a partir de quatro estudos de caso

também facilita os processos de aprendizado interativo. Mesmo que se reconheça a importância da proximidade geográfica, as empresas podem estabelecer projetos de colaboração com universidades mais distantes. Nesses casos, a elevada capacidade de absorção da empresa e a proximidade cognitiva entre os atores foram capazes de transpor os benefícios da proximidade geográfica.

Por fim, deve-se mencionar a importância do acesso ao financiamento público, especialmente por meio de programas de subvenção. Todas as empresas analisadas foram beneficiárias de programas públicos de apoio à inovação: os esquemas de financiamento público à inovação foram importantes catalizadores dos projetos de cooperação entre as empresas e a universidade. Os problemas típicos das pequenas empresas, relacionados com a escassez de recursos físicos, financeiros e humanos, são, em grande parte, suplantados pela existência desses programas de apoio, os quais permitem o rompimento da barreira de recursos financeiros para o investimento em projetos colaborativos de inovação de alto risco, o acesso a recursos humanos da universidade para apoio aos projetos colaborativos das empresas e à infraestrutura laboratorial e de pesquisa da universidade.

Os resultados alcançados traduzem-se em implicações de políticas. As políticas de apoio à inovação em pequenas empresas devem considerar o papel desses direcionadores da colaboração entre as pequenas empresas e a universidade. Em especial, é importante que as políticas incorporem a necessidade de elevação da capacidade de absorção das pequenas empresas, por demonstrar um fator de sucesso muito importante para a colaboração entre as empresas e para os resultados da colaboração em si.

\begin{abstract}
This paper presents new evidence in the subject of the cooperation between universities and research institutes and firms, by investigating the main drivers of the interaction between university and small and medium enterprises. To do that, it explores the results of four case studies of cooperation between small firms with universities in Brazil. Main findings show that the firms' absorptive capacity, the existence of previous linkages, and geographic and cognitive proximities are important drivers of the collaboration. In addition, results reveal that high absorptive capacity and high cognitive proximity between agents can foster cooperation at greater geographical distance. Moreover, findings also show the importance of public funding for cooperation, especially when they involve greater complexity and risk projects.
\end{abstract}

Keywords: University-industry cooperation; small and medium enterprises; driving factors; knowledge and innovation

JEL: O31, O32, O33, L26

Econômica-Niterói, v. 20, n. 2, p. 85-106. Dezembro, 2018 


\section{Referências bibliográficas}

ACS, Z. J.; AUDRETSCH, D. B.; FELDMAN, M. P. R \&amp; D Spillovers and Recipient Firm Size. The Review of Economics and Statistics, 1994. ISSN 00346535.

ACS, Z. J.; AUDRETSCH, D. B.; FELDMAN, M. P. R\&D spillovers and innovative activity. Managerial and Decision Economics, 1994. ISSN 10991468.

ALLEN, T. J. T. J. Managing the flow of technology: technology transfer and the dissemination of technological information within the $R \&$ amp; D organization. [S.1.]: [MIT Press], 1977. 320 p. ISBN 9780262010481.

ALVES-FILHO, M. Olho eletrônico obtém dectecção automática de parasitos intestinais. Campinas: [s.n.], 2007. 5 p.

AUDRETSCH, D. B.; VIVARELLI, M. Small firms and R\&amp;D spillovers : Evidence from Italy. Revue d'économie industrielle, Persée - Portail des revues scientifiques en SHS, v. 67, n. 1, p. 225-237, 1994. ISSN 0154-3229. Disponível em: $<$ https://www.persee.fr/doc/rei\_0154-3229\_1994\_num\67\_1\1520>.

BISHOP, K.; D'ESTE, P.; NEELY, A. Gaining from interactions with universities: Multiple methods for nurturing absorptive capacity. Research Policy, v. 40, n. 1, p. 30-40, 2011.

BOSCHMA, R. Proximity and Innovation: A Critical Assessment. Regional Studies, Taylor \& Francis Group, v. 39, n. 1, p. 61-74, 2 2005. ISSN 0034-3404. Disponível em: $<$ http://www.tandfonline.com/doi/abs/10.1080/0034340052000320887>.

BOSCHMA, R. A.; LAMBOOY, J. G. Evolutionary economics and economic geography. Journal of Evolutionary Economics, Springer-Verlag, v. 9, n. 4, p. 411-429, 121999. ISSN 0936-9937. Disponível em: <http://link.springer.com/10.1007/s001910050089>.

BROSTRÖM, A. Working with distant researchers-Distance and content in university-industry interaction. Research Policy, v. 39, n. 10, p. 1311-1320, 2010. Disponível em: <https://www.sciencedirect.com/journal/research-policy/vol/39/issue/ $10>$.

BRUNEEL, J.; D'ESTE, P.; SALTER, A. Investigating the factors that diminish the barriers to university-industry collaboration. Research Policy, v. 39, n. 7, p. 858-868, 2010. Disponível em: <https://www.sciencedirect.com/journal/research-policy/vol/39/ issue/7>.

CARDAMONE, P.; PUPO, V. R\&amp;D Cooperation Between Firms And Universities. Some Evidence In Five European Countries. Working Papers, Università della Calabria, 
104 Fatores direcionadores da cooperação de pequenas e médias empresas com a universidade: evidências a partir de quatro estudos de caso

Dipartimento di Economia, Statistica e Finanza "Giovanni Anania- DESF, 2015. Disponível em: <https://ideas.repec.org/p/clb/wpaper/201501.html>.

CARRIJO, M. d. C.; BOTELHO, M. d. R. A. Cooperação e inovação: uma análise dos resultados do Programa de Apoio à Pesquisa em Empresas (Pappe). Revista Brasileira de Inovação, v. 12, n. 2, p. 417, 9 2013. ISSN 2178-2822. Disponível em: <https://periodicos.sbu.unicamp.br/ojs/index.php/rbi/article/view/8649067>.

COHEN, W. M.; LEVINTHAL, D. A. Absorptive Capacity: A New Perspective on Learning and Innovation. Administrative Science Quarterly, Sage Publications, Inc.Johnson Graduate School of Management, Cornell University, v. 35, n. 1, p. 128, 3 1990. ISSN 00018392. Disponível em: <https://www.jstor.org/stable/2393553?origin= crossref $>$.

DREJER, I.; ØSTERGAARD, C. R. Exploring determinants of firms' collaboration with specific universities: employee-driven relations and geographical proximity. Regional Studies, Routledge, v. 51, n. 8, p. 1192-1205, 8 2017. ISSN 0034-3404. Disponível em: <https://www.tandfonline.com/doi/full/10.1080/00343404.2017.1281389>.

FONTANA, R.; GEUNA, A.; MATT, M. Firm size and openness: the driving forces of universityindustry collaboration. In: SPRU Working Paper Series. Sussex: Science and Technology Policy Research, University of Sussex, 2003.

FUENTES, C. D.; DUTRÉNIT, G. Best channels of academia-industry interaction for long-term benefit. Research Policy, v. 41, n. 9, p. 1666-1682, 2012. Disponível em: $<$ https://www.sciencedirect.com/journal/research-policy/vol/41/issue/9>.

GARCIA, R. et al. Looking at both sides: how specific characteristics of academic research groups and firms affect the geographical distance of university-industry linkages. Regional Studies, Regional Science, Routledge, v. 2, n. 1, p. 518-534, 12015. ISSN 2168-1376. Disponível em: <https://www.tandfonline.com/doi/full/10.1080/ 21681376.2015.1099464>.

GARCIA, R. et al. Os efeitos da proximidade geográfica para o estímulo da interação universidade-empresa. Revista de Economia, v. 37, n. 4, 12 2011. ISSN 2316-9397. Disponível em: <http://revistas.ufpr.br/economia/article/view/27687>.

GARCIA, R. e. a. An analysis of the relation between geographical and cognitive proximity in university-industry linkages. In: 44 Encontro Nacional de Economia. Foz do Iguaçu: Anpec, 2016.

GOMES, J.; HOSHINO-SHIMIZU, S.; FALCAO, A. Recentes avanços tecnológicos no exame parasitológico de fezes. BIO FARMA - Revista Técnico-Científica de Farmácia, Bioquímica e Análises Clínicas e Toxicológicas, v. 3, n. 6, p. 44-53, 2008.

Econômica-Niterói, v. 20, n. 2, p. 85-106. Dezembro, 2018 
INOVA UNICAMP. Relatório de Atividades 2011. Campinas, 2011.

LAURSEN, K.; REICHSTEIN, T.; SALTER, A. Exploring the Effect of Geographical Proximity and University Quality on University-Industry Collaboration in the United Kingdom. Regional Studies, Routledge, v. 45, n. 4, p. 507-523, 4 2011. ISSN 0034-3404. Disponível em: <http://www.tandfonline.com/doi/full/10.1080/00343400903401618>.

LAURSEN, K.; SALTER, A. Searching high and low: what types of firms use universities as a source of innovation? Research Policy, North-Holland, v. 33, n. 8, p. 1201-1215, 10 2004. ISSN 0048-7333. Disponível em: <https: //www.sciencedirect.com/science/article/abs/pii/S0048733304001003>.

LEMOS, D. d. C.; CARIO, S. A. F. Análise da interação universidade-empresa para o desenvolvimento inovativo a partir da perspectiva teórica institucionalista-evolucionária. Revista Brasileira de Inovação, v. 14, n. 2, p. 361, 9 2015. ISSN 2178-2822. Disponível em: <https://periodicos.sbu.unicamp.br/ojs/index.php/rbi/article/view/8649112>.

LINK, A. N.; REES, J. Firm size, university based research, and the returns to R\&amp;D. Small Business Economics, Kluwer Academic Publishers, v. 2, n. 1, p. 25-31, 1990. Disponível em: <http://link.springer.com/10.1007/BF00389891>.

MOHNEN, P.; HOAREAU, C. What type of enterprise forges close links with universities and government labs? Evidence from CIS 2. Managerial and Decision Economics, Wiley-Blackwell, v. 24, n. 2-3, p. 133-145, 3 2003. ISSN 0143-6570. Disponível em: <http://doi.wiley.com/10.1002/mde.1086>.

MORA-VALENTIN, E. M.; MONTORO-SANCHEZ, A.; GUERRAS-MARTIN, L. A. Determining factors in the success of R\&amp;D cooperative agreements between firms and research organizations. Research Policy, v. 33, n. 1, p. 17-40, 1 2004. ISSN 00487333. Disponível em: <http://linkinghub.elsevier.com/retrieve/pii/ S0048733303000878>.

NELSON, R. R.; WINTER, S. G. Uma Teoria Evolucionária da Mudança Econômica. Campinas: Unicamp, 2005. 632 p. ISBN 8526807129. Disponível em: <https: //www.saraiva.com.br/uma-teoria-evolucionaria-da-mudanca-economica-194603.html>.

PADTEC. Relatório de Administração. Campinas, 2017.

RAPINI, M. S.; OLIVEIRA, V. P. d.; SILVA-NETO, F. C. d. C. e. A natureza do financiamento influencia na interação universidade-empresa no Brasil? Revista Brasileira de Inovação, v. 13, n. 1, p. 77, 12 2013. Disponível em: <https: //periodicos.sbu.unicamp.br/ojs/index.php/rbi/article/view/8649072>.

SALLES-FILHO, S. Quanto vale o investimento em ciência, tecnologia e inovação? Campinas: [s.n.], 2011. 
106 Fatores direcionadores da cooperação de pequenas e médias empresas com a universidade: evidências a partir de quatro estudos de caso

SALLES-FILHO, S. et al. Avaliação de impactos da Lei de Informática: uma análise da política industrial e de incentivo à inovação no setor de TICs brasileiro. Revista Brasileira de Inovação, v. 11, n. 0, p. 191, 7 2012. Disponível em: $<$ https://periodicos.sbu.unicamp.br/ojs/index.php/rbi/article/view/8649041>.

SCHARTINGER, D. et al. Knowledge interactions between universities and industry in Austria: sectoral patterns and determinants. Research Policy, NorthHolland, v. 31, n. 3, p. 303-328, 3 2002. ISSN 0048-7333. Disponível em: $<$ https://www.sciencedirect.com/science/article/abs/pii/S0048733301001111>.

TEIXEIRA, A. L. d. S. et al. Dimensões da capacidade de absorção, qualificação da mão de obra, P\&amp;D e desempenho inovativo. Revista Brasileira de Inovação, v. 15, n. 1, p. 139, 3 2016. Disponível em: <https://periodicos.sbu.unicamp.br/ojs/index.php/rbi/ article/view/8649123>.

TEIXEIRA, C.; MENEZES, J. H. Resultados do RHAE Pesquisador na Empresa. In: XV CONGRESSO DA ASSOCIAÇÃO LATINO-IBEROAMERICANA DE GESTÃO DE TECNOLOGIA. Porto: XV CONGRESSO DA ASSOCIAÇÃO LATINOIBEROAMERICANA DE GESTÃO DE TECNOLOGIA., 2013. 